\title{
Glucagon-like peptide 1 improves insulin resistance in vitro through anti-inflammation of macrophages
}

\author{
C. Guo, T. Huang, A. Chen, X. Chen, L. Wang, F. Shen and X. Gu
}

Department of Endocrinology, The First Affiliated Hospital of Wenzhou Medical University, Wenzhou, Zhejiang, China

\begin{abstract}
Glucagon-like peptide 1 (GLP-1), a kind of gut hormone, is used in the treatment of type 2 diabetes (T2D). Emerging evidence indicates that GLP-1 has anti-inflammatory activity. Chronic inflammation in the adipose tissue of obese individuals is a cause of insulin resistance and T2D. We hypothesized that GLP-1 analogue therapy in patients with T2D could suppress the inflammatory response of macrophages, and therefore inhibit insulin resistance. Our results showed that GLP-1 agonist (exendin-4) not only attenuated macrophage infiltration, but also inhibited the macrophage secretion of inflammatory cytokines including TNF- $\beta$, IL-6, and IL-1 $\beta$. Furthermore, we observed that lipopolysaccharide (LPS)-induced macrophage conditioned media could impair insulinstimulated glucose uptake. This effect was compensated by treatment with the conditioned media from macrophages treated with the combination of LPS and exendin-4. It was also observed that exendin-4 directly inhibited the activation of NF-kB in macrophages. In conclusion, our results indicated that GLP-1 improved inflammatory macrophage-derived insulin resistance by inhibiting NF- $\mathrm{KB}$ pathway and secretion of inflammatory cytokines in macrophages. Furthermore, our observations suggested that the antiinflammatory effect of GLP-1 on macrophages can contribute to GLP-1 analogue therapy of T2D.
\end{abstract}

Key words: Diabetes; Glucagon-like peptide; Macrophage infiltration; Adipose inflammation; Insulin resistance

\section{Introduction}

Obesity and type 2 diabetes (T2D) are considered chronic pro-inflammatory diseases (1). Adipose tissue macrophages (ATMs) produce inflammatory cytokines and play an important role in such chronic inflammatory responses $(2,3)$. Macrophages that are mostly polarized have either an M1 or an M2 phenotype (4). In general, the activation status of M1 or M2 phenotype are influenced by local microenvironments $(5,6)$. Th1 cytokines (such as IFN- $\gamma$ ) induce activation of M1 macrophages, which produce inflammatory mediators. Th2 cytokines (such as IL-4 and IL-13) induce the alternative activation of M2 macrophages, which regulate anti-inflammatory responses. Accumulated evidence has proven that macrophage polarization plays a critical role in the development of T2D. It is considered that the imbalance in the M1/M2 macrophage ratio leads to chronic inflammation in adipose tissue of T2D patients (1). The majority of ATMs in lean individuals exhibit an anti-inflammatory M2 polarity. Accumulation of M1 macrophages in obesity individuals are associated with insulin resistance $(1,7,8)$.

Obesity-related insulin resistance plays a critical role in the cause and development of diabetic pathophysiology (9). The infiltration of macrophages into adipose tissue is the initial event of inflammation in the adipose tissues (10). M2 macrophages produce anti-inflammatory cytokines, such as IL-10. It is reported that M2 macrophages can increase insulin-dependent glucose uptake in adipocytes of lean people (11). M1 macrophages, which represent a pro-inflammatory state, can secrete inflammatory cytokines such as tumor necrosis factor (TNF)- $\alpha$, interleukin- 6 (IL-6) and IL-1 $\beta$ that interfere with insulin signaling, and cause adipocyte dysfunction and insulin resistance $(4,10)$.

NF-кB pathway plays a key role in inflammation by its ability to induce transcription of pro-inflammatory genes (12). The NF-кB family is formed by several members,

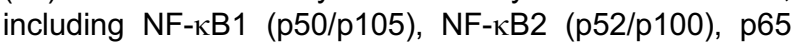
(RelA), RelB, and c-Rel (13). The activity of NF-kB is regulated by inhibitors of $\kappa B(I \kappa B)$ proteins and $I_{\kappa} B$ kinase (IKK). NF-KB is located in the cytoplasm in an inactive form, which associates with $1 \kappa B$. The most important form of $I \kappa B$ is $I \kappa B \alpha, I \kappa B \beta$, and $I \kappa B \in$. Phosphorylation of $I \kappa B$ that is mediated by IKK is an important step in NF- $\mathrm{NB}$, which causes dissociation of $\mathrm{NF}-\kappa \mathrm{B}$ and $\mathrm{I} \kappa \mathrm{B}$ and its translocation into nuclei (14). As one of the most important regulators of pro-inflammatory gene expression, NF- $\kappa \mathrm{B}$ regulates the synthesis of many inflammatory cytokines including TNF- $\alpha$, IL-1 $\beta$, IL-6 and IL-8 $(14,15)$.

Glucagon-like peptide-1 (GLP-1), a kind of incretin hormone, is secreted by intestinal $L$ cells in response to

Correspondence: X. Gu: <xuemeigu1@21cn.com> 
nutrients. Then, GLP-1 stimulates the release of insulin from pancreatic $\beta$-cells (16). It has been reported that the secretion of GLP-1 decreases in patients with T2D (17). Now, the GLP-1 analogues have been used in combination with insulin for patients with T2D. It has also been reported that a GLP-1 agonist plays an anti-inflammatory function in cultured human macrophages (18). Moreover, GLP can inhibit adipose tissue macrophage infiltration and inflammation in an obese mouse model of diabetes (19). However, whether GLP-1 affects insulin resistance by suppressing macrophage inflammation is not clear.

In the present study, we evaluated the effects of exendin-4, a kind of GLP-1 analogue, in the infiltration of macrophages, and in the secretion of inflammatory cytokines including TNF- $\alpha$, IL- 6 , and IL- $1 \beta$ by inactivation of NF-kB pathway. The inhibitory effect of GLP-1 on macrophage inflammation further prevents the impairment of insulin sensitivity induced by lipopolysaccharide (LPS)stimulated inflammation. Our results that GLP-1 improved insulin resistance by anti-inflammation of macrophages provide a new biological mechanism for the clinical therapeutics of T2D.

\section{Material and Methods}

\section{Cell culture and cell transfection}

Mouse peritoneal macrophages were prepared, isolated and cultured as described previously (20). The mouse macrophage cell line (RAW264) and mouse preadipocyte (3T3-L1s) were obtained from Shanghai Institute of Chinese Academy of Sciences (China). RAW264 cells were maintained in DMEM (Gibco, USA), supplemented with $10 \%$ fetal bovine serum at $37^{\circ} \mathrm{C}$ in a humidity incubator within $5 \% \mathrm{CO}_{2}$. $3 \mathrm{~T} 3-\mathrm{L} 1$ cells were cultured in DMEM, supplemented with $10 \%$ calf serum at $37^{\circ} \mathrm{C}$ in a humidity incubator within $5 \% \mathrm{CO}_{2}$. RAW264 cells were grown in 6 -well plates with a $75 \%$ confluence at $24 \mathrm{~h}$ before transfection. Cell transfection was carried out using lipofectamine2000 (Invitrogen, USA) according to the manufacturer's description. RAW264 cells and mouse peritoneal macrophages were transfected with siRNA of GLP-1 receptor (GLP$1 \mathrm{R})$ and scramble siRNA. The sequences of siRNA were described in previous studies $(21,22)$ : GLP-1R forward, 5'-AUA AUG AGC CAG UAG UUC AUG UUGG-3' and reverse, 5'-CCA ACA UGA ACU ACU GGC UCA UUAU-3'; negative control (scramble) forward, 5'-UUC UCC GAA CGU GUC ACG UTT-3'; reverse, 5'-ACG UGA CAC GUU CGG AGA ATT-3'. These sequences were synthesized by GenePharma Co. (China).

\section{Protein extraction and western blot}

Protein extraction and western blot were performed as previously described (23). Briefly, total proteins were extracted from RAW264 cells and mouse peritoneal macrophages using a RIPA lysis buffer (Beyotime Biotech Inc., China) supplemented with Complete EDTA-free protease inhibitor cocktail tablets (Roche, USA) according to the manufacturer's instructions. Total protein concentrations were assayed with the BCA protein assay kit (Applygen, China). The protein concentration was diluted to $2 \mu \mathrm{g} / \mu \mathrm{L}$ in every sample. Approximately $40 \mu \mathrm{g}$ of protein in every sample were fractionated by gel electrophoresis, and transferred to a polyvinylidene fluoride membrane (Millipore, USA), blocked with $5 \%$ skim milk for $1 \mathrm{~h}$, and then incubated with primary antibodies including anti-

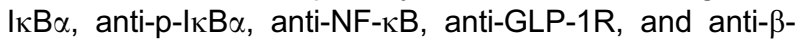
actin (Santa Cruz, USA) at $4^{\circ} \mathrm{C}$ overnight. The membrane was next incubated with horseradish peroxidase-conjugated secondary antibodies for $1 \mathrm{~h}$ after three washes with TBST. Signals were tested by enhanced chemiluminescence detection reagent (Thermo Scientific, USA) and protein expressions were calculated by normalization to $\beta$-actin. All detection reactions were repeated three times. Western blots showed in the figures are representative of three independent experiments.

\section{RNA extraction and qPCR}

Total RNA was isolated from RAW264 cells using Trizol (Invitrogen) under the manufacturer's instructions and then cDNA was generated from RNA using a First Strand cDNA Synthesis Kit (Fermentas, USA). Quantitative real-time PCR (qRT-PCR) was performed using $2 \times$ TransStart Green qPCR SuperMix (TransGen Biotech Co., China) on an $\mathrm{ABI} 7300$ instrument as previously described (24). mRNA levels were normalized to the level of $\beta$-actin. PCRs were performed in duplicates, and error bars in the charts represent the corresponding standard deviations. The primers used to detect mouse GLP-1R (22) were forward: 5'-TTG GGG TGA ACT TCC TCA TC-3', reverse: 5'-CTT GGC AAG TCT GCA TTT GA-3'; $\beta$-actin (25) forward: $5^{\prime}$-GCC AAC CGT GAA AAG ATG ACC-3', and reverse: 5'-CCC TCG TAG ATG GGC ACA GT-3'.

\section{Transwell assays}

Transwell migration assay of macrophage was performed as previously described (25). Briefly, $2 \times 10^{5}$ RAW264 cells or mouse peritoneal macrophages transfected with scramble or GLP-1R siRNAs were supplemented with $100 \mu \mathrm{L}$ serum-free medium and placed in the upper chamber with 8-mm pore size (BD Bioscience, USA) for migration assays, while $600 \mu \mathrm{L}$ serum-free medium with or without LPS $(200 \mathrm{ng} / \mathrm{mL})$ were placed in the lower chamber. One of LPS groups was added with exendin-4 into the upper chamber. After incubation for $24 \mathrm{~h}$ at $37^{\circ} \mathrm{C}$, the cells in the upper membrane were discarded and cells on the lower membrane were fixed using $95 \%$ ethanol and stained with crystal violet (Beyotime). Next, five random fields were counted. Each experiment was performed in triplicate. Migrated cell number was measured with Image-Pro Plus 6.0 software (Media Cybernetics, USA). 
Macrophage-conditioned media (CM) preparation and inflammatory factors detection

Seventy to eighty percent confluence of RAW264 cells or mouse peritoneal macrophages in 6-well plates were starved with serum-free medium, or serum-free DMEM containing LPS $(200 \mathrm{ng} / \mathrm{mL})$ with or without exendin-4 (2.5 nM; Sigma, USA) overnight. Then the cells were washed for three times using PBS, and incubated in serum-free medium for $24 \mathrm{~h}$. The cells cultured in conditioned media were collected, centrifuged at $500 \mathrm{~g}$ for $5 \mathrm{~min}$ at $4^{\circ} \mathrm{C}$, filtered through a $0.22-\mu \mathrm{m}$ syringe filter, and stored at $4^{\circ} \mathrm{C}$ before being used for the experiments. RAW264 cells or mouse peritoneal macrophages starved with serum-free medium were defined as macrophageconditioned media (CM). CM from macrophages treated with serum-free DMEM containing LPS with or without exendin-4 (2.5 nM; Sigma) were defined as CM-LPS-Ex4 or CM-LPS, respectively.

The levels of TNF- $\alpha$, IL- 6 , and IL- $1 \beta$ in conditioned media were investigated using enzyme-linked immunosorbent assay (ELISA). The concentration of these factors was measured using a Human Quantikine ELISA kit (R\&D Systems, USA), according to the manufacturer's instructions.

\section{Insulin-stimulated glucose uptake}

3T3-L1 adipocytes were used for determining insulinstimulated glucose uptake as previously described (26). Briefly, the 3T3-L1 preadipocytes were differentiated into adipocytes as described in a previous report (25). After differentiation, the medium was switched to low-glucose DMEM containing $0.3 \%$ bovine serum albumin (BSA) alone (control group) or with CM, CM-LPS, or CM-LPSEx4 and incubated at $37^{\circ} \mathrm{C}$ for $16 \mathrm{~h}$. Then, the medium was switched to a $\mathrm{KRBH}$ buffer containing $10 \mathrm{nM}$ of insulin with or without vehicles (DMSO), and with methanol and water extracts, and further incubated at $37^{\circ} \mathrm{C}$ for $30 \mathrm{~min}$. After incubation, $0.1 \mathrm{ICi}$ 2-deoxy-D-[ $\left.{ }^{3} \mathrm{H}\right]$ glucose was added into the $\mathrm{KRBH}$ buffer for $10 \mathrm{~min}$. At the end of the incubation, the buffer was removed and the cells were washed three times with ice-cold PBS. The radioactivity of ${ }^{3} \mathrm{H}$ was counted using a Wallac Liquid Scintillation Counter (USA) to determine glucose uptake. Non-specific glucose uptake was measured in cells treated with vehicles and with methanol and water extracts without insulin.

\section{Statistical analyses}

Data are reported as means $\pm S D$. Student's $t$-test was performed to assess differences between two groups. One-way ANOVA was conducted to assess differences among multiple groups. All statistical calculations were carried out using SPSS 19.0 software (USA) and P $<0.05$ was considered statistically significant.

\section{Results}

\section{GLP-1R knockdown with siRNA in macrophages}

To evaluate the function of GLP-1, siRNA was used to knockdown GLP-1R in macrophages. Then, the knockdown effect of GLP-1R siRNA was detected by qPCR and western blot. The results showed that the expression levels of GLP-1R were significantly decreased (Figure $1 A-C)$. Therefore, siRNA for GLP-1R can be used to analyze the function of GLP in macrophages.

\section{Exendin-4 inhibited the migration of macrophages}

The infiltration of macrophages initiates low-grade inflammation in the adipose tissues, which is an important factor in the development of diabetes (27). To determine whether GLP affects macrophage infiltration, we treated RAW264 cells or mouse peritoneal macrophages with LPS, and tested whether LPS treatment-induced macrophages migration was inhibited by exendin- 4 that is a long-acting potent agonist of GLP-1R (28). Our results showed that LPS led to an increased transwell migration of RAW264 cells and mouse peritoneal macrophages, and this effect was reversed by exendin-4 treatment
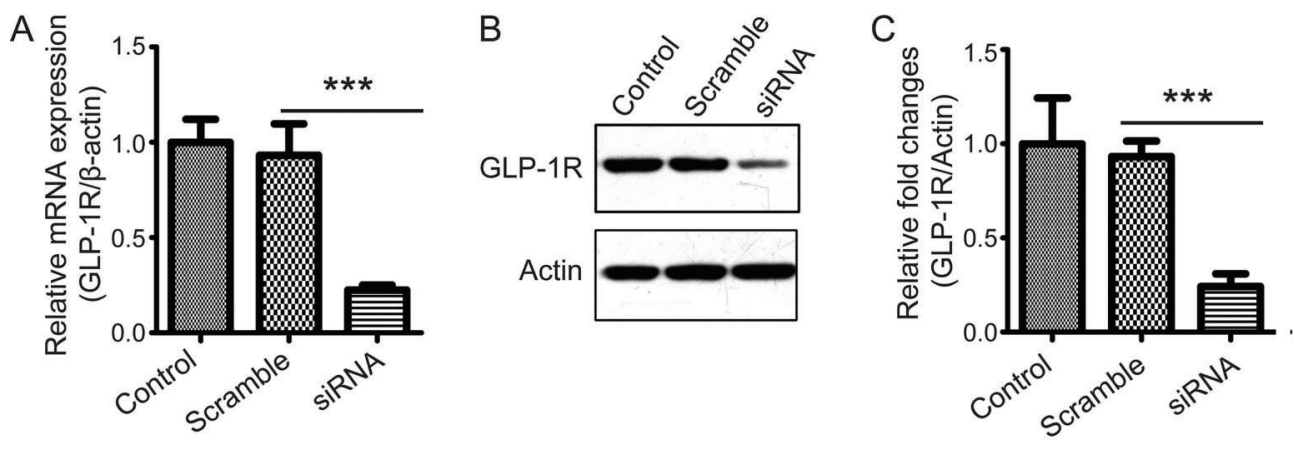

Figure 1. GLP-1R knockdown in macrophages using siRNA. RAW264 cells were transfected with siRNA of GLP-1 receptor (GLP-1R) and scramble siRNA for $72 \mathrm{~h}$. A, The mRNA levels of GLP-1R normalized to $\beta$-actin were assessed by qPCR. Data are reported as relative values compared to the control group $(n=6)$. $B$, Protein levels of GLP-1R assessed by western blot. $C$, Relative fold changes by western blot. The results are representative of three independent experiments. Data are reported as means $\pm S D$. ${ }^{* \star *} P<0.001$ (ANOVA). 

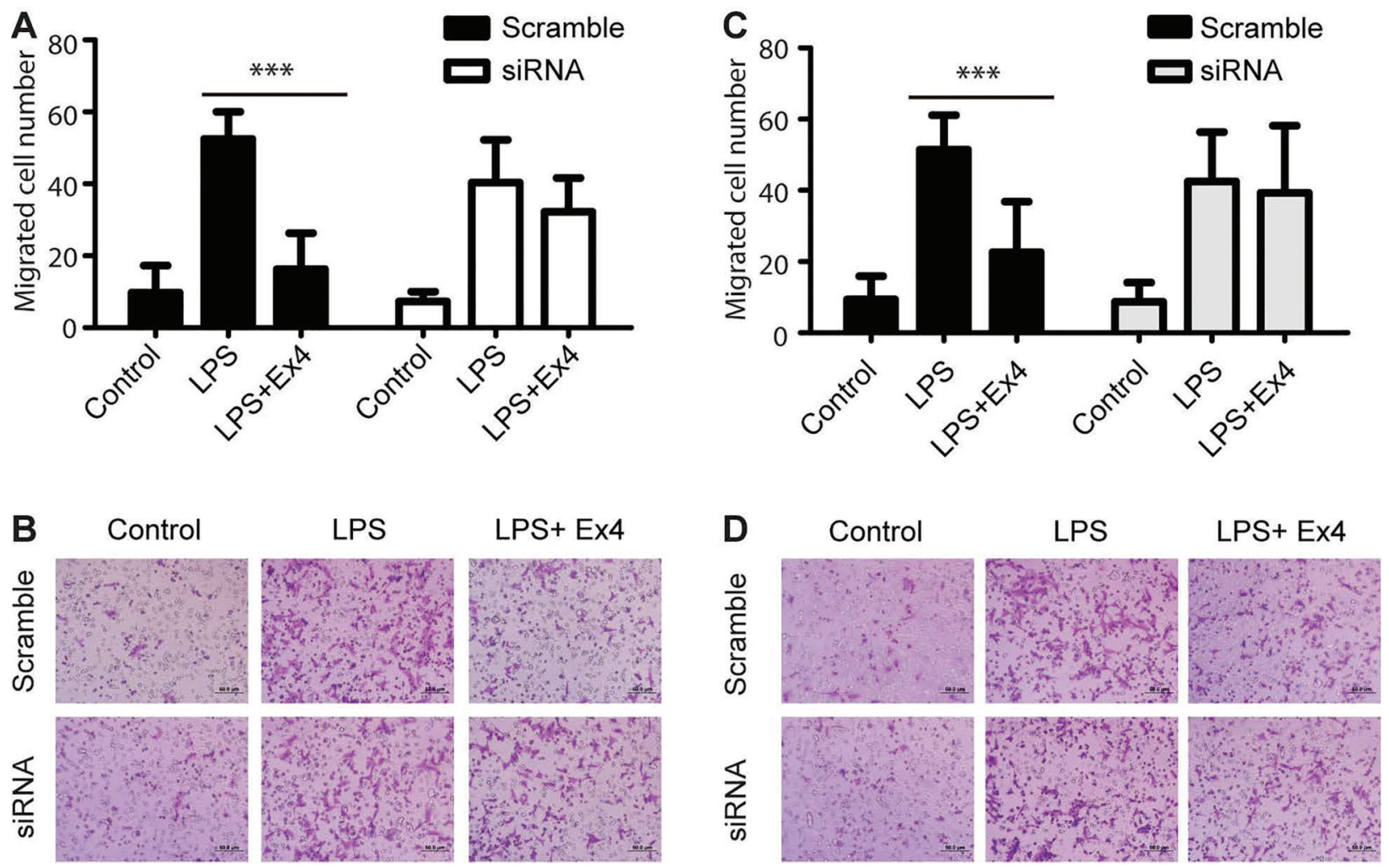

Figure 2. Effect of exendin-4 (Ex4) on the migration of macrophages and corresponding photomicrographs (bar: $50 \mu \mathrm{m})$. RAW264 cells $(A$ and $B)$ or mouse peritoneal macrophages $(C$ and $D)$ were treated with lipopolysaccharide (LPS) or a combination of LPS and exendin-4, and then transwell migration was performed for $24 \mathrm{~h}$. Data are reported as means $\pm \mathrm{SD}$. ${ }^{* *} \mathrm{P}<0.001$ (ANOVA).

(Figure 2A-D). Moreover, knocking down of GLP-1R using siRNA could rescue the inhibitory effect of exendin-4 on macrophage migration (Figure $2 A-D$ ). These results suggest that GLP can suppress LPS-induced macrophage infiltration.

\section{Exendin-4 inhibited the secretion of inflammatory factors}

Inflammatory cytokines such as TNF- $\alpha$, IL-6, and IL-1 $\beta$ can cause adipocyte dysfunction, which is involved in the development of diabetes. To study the effect of GLP-1 on the macrophage-secreted inflammatory cytokines, we detected the levels of TNF- $\alpha$, IL- 6 , and IL- $1 \beta$ in RAW264 cells and mouse peritoneal macrophages after treatment with exendin-4. As showed in Figure $3 A$ and $B, L P S$ treatment induced the upregulation of TNF- $\alpha$, IL- 6 , and IL-1 $\beta$ in RAW264 and mouse peritoneal macrophages, which could be rescued by exendin- 4 treatment. In addition, knocking down GLP-1R in RAW264 cells and mouse peritoneal macrophages using siRNA could reverse the inhibitory effect of exendin- 4 on secretion of TNF- $\alpha$, IL- 6 , and IL-1 $\beta$ (Figure $3 A$ and $B$ ). Taken together, all of these results suggest that GLP could reduce LPS-induced inflammatory cytokines secretion of macrophages.

\section{Exendin-4 increased insulin-stimulated glucose} uptake by targeting inflammatory macrophages

It was reported that adipose tissue inflammation in the prediabetic state is related to increased insulin resistance $(29,30)$. To explore the effect of exendin-4 on macrophagesecreted inflammatory cytokines mediated insulin resistance, we tested the insulin-stimulated glucose uptake using 3T3L1 adipocytes which were incubated with LPS or exendin4-treated macrophage CM from RAW264 cells or mouse peritoneal macrophages. Our results showed that macrophage $\mathrm{CM}$ and LPS-treated macrophage $\mathrm{CM}$ reduced insulin-stimulated glucose uptake (Figure 4A and B). Moreover, CM from exendin-4 and LPS-treated macrophages could rescue the inhibitory effect of CM-LPS on insulinstimulated glucose uptake, which could be abrogated by GLP-1R knockdown in macrophages (Figure 4A and B). Taken together, these results suggest that GLP inhibited macrophage-secreted inflammatory factors and induced insulin resistance in vitro.

\section{Exendin-4 inhibited activation of NF- $\kappa \mathrm{B}$ in macrophages}

All of the above results suggested that GLP inhibited insulin resistance by preventing the inflammation response 

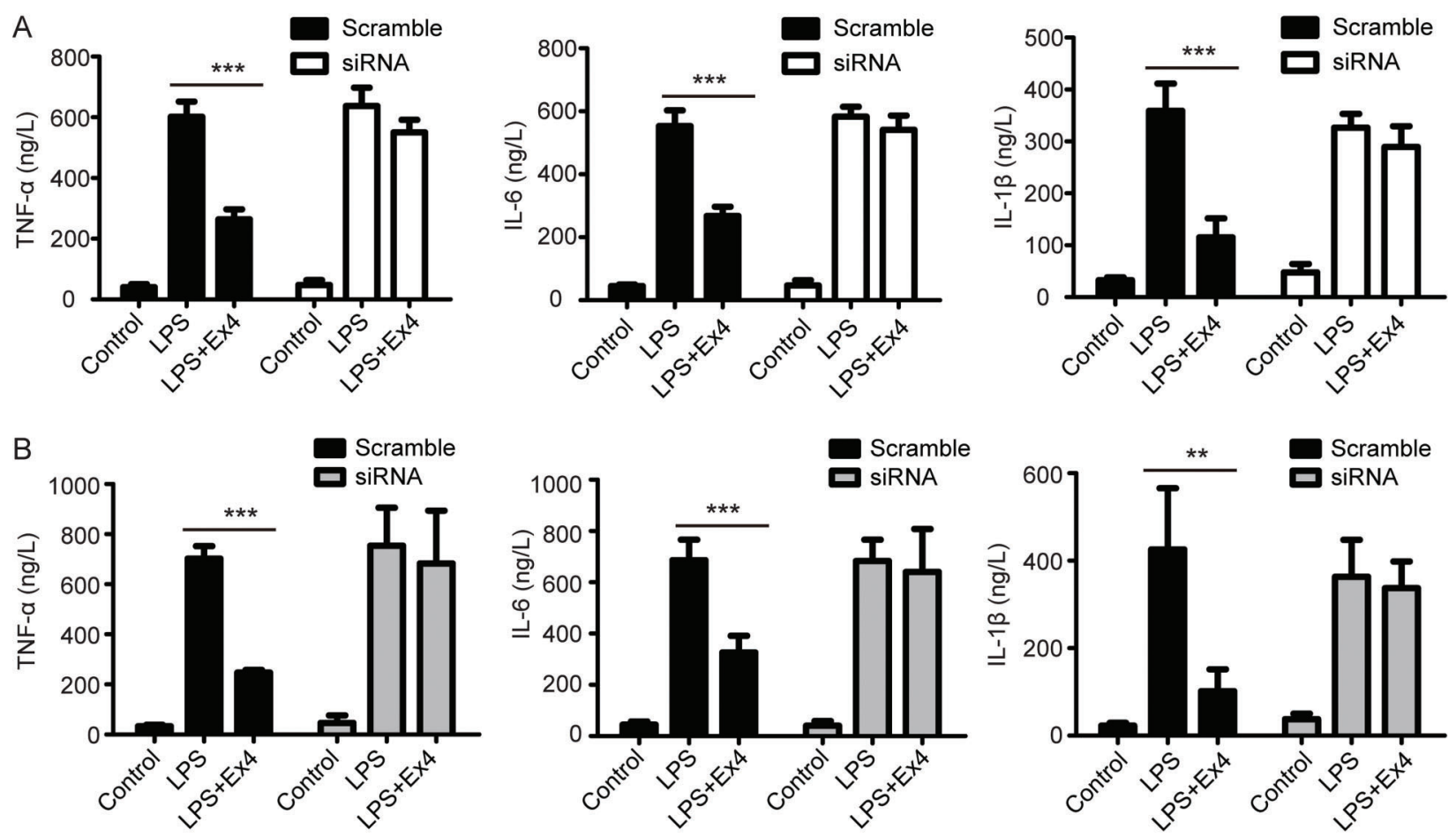

Figure 3. Effect of exendin-4 (Ex4) on the secretion of inflammatory macrophage cytokines. RAW264 cells $(A)$ or mouse peritoneal macrophages $(B)$ were treated with lipopolysaccharide (LPS) or a combination of LPS and Ex4 overnight, and then incubated in serumfree medium for $24 \mathrm{~h}$. The secretion of TNF- $\alpha$, IL-6, and IL-1 $\beta$ was evaluated using enzyme-linked immunosorbent assay (ELISA). Data are reported as means $\pm \mathrm{SD}$. ${ }^{* *} \mathrm{P}<0.01 ;{ }^{* * *} \mathrm{P}<0.001$ (ANOVA).
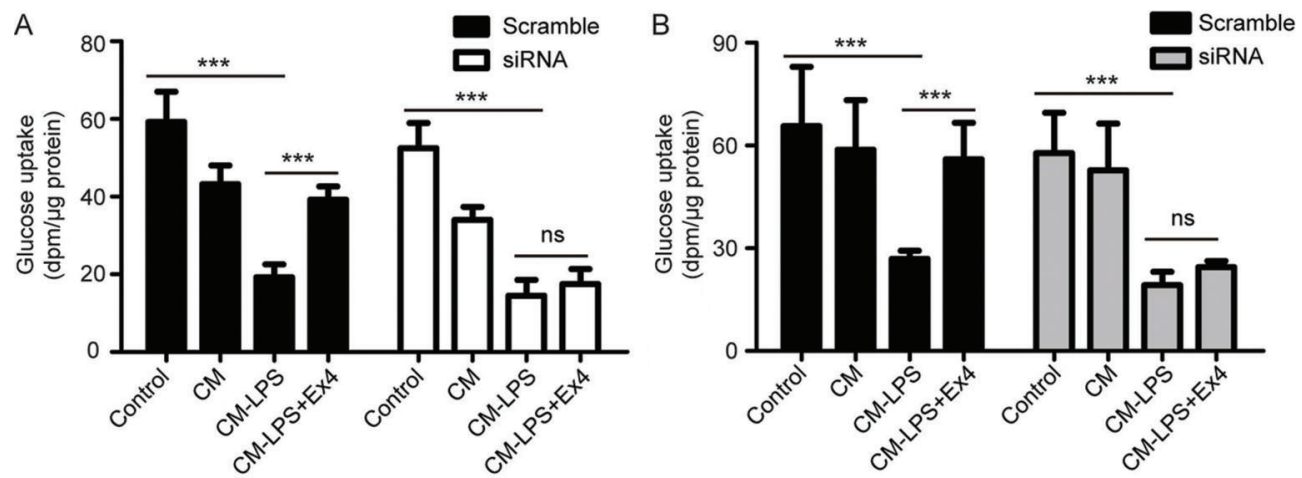

Figure 4. Effect of exendin-4 (Ex4)-treated macrophage conditioned media (CM) on insulin-stimulated glucose uptake. 3T3-L1 adipocytes were untreated (control group) or treated with CM, CM-lipopolysaccharide (LPS), or CM-LPS-Ex4 from RAW264 cells ( $A$ ) or mouse peritoneal macrophages $(B)$. The results are representative of three independent experiments. Data are reported as means \pm SD. ${ }^{* * *} \mathrm{P}<0.001$ (ANOVA). ns: non-significant.

of macrophages. It was reported that activation of NF- $\kappa B$ is involved in the secretion of inflammatory cytokines. To investigate the mechanism of GLP-suppressed inflammation, we examine the expression and phosphorylation level of $\mathrm{I} \kappa \mathrm{B} \alpha$, and the nuclear translocation of NF- $\mathrm{KB}$ in RAW246 cells and mouse peritoneal macrophages. Our results showed that LPS did not affect the expression levels of
$I_{\kappa} \mathrm{B} \alpha$, but increased the phosphorylation of $I_{\kappa} \mathrm{B} \alpha$ and the nuclear translocation of NF- $\mathrm{KB}$ (Figures 5 and 6), which suggests that LPS could activate the transcription factor NF- $\mathrm{KB}$. Furthermore, we found that exendin-4 treatment could reverse the activation of NF- $\mathrm{BB}$ induced by LPS (Figures 5 and 6). Thus, we infer that GLP likely inhibits the secretion of inflammatory cytokines by inactivation of NF-kB pathway. 

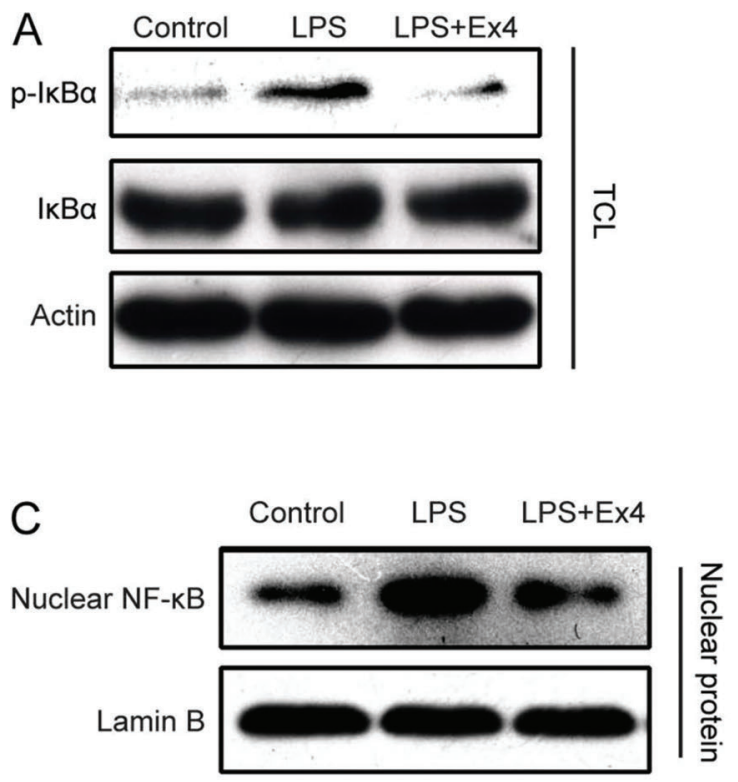
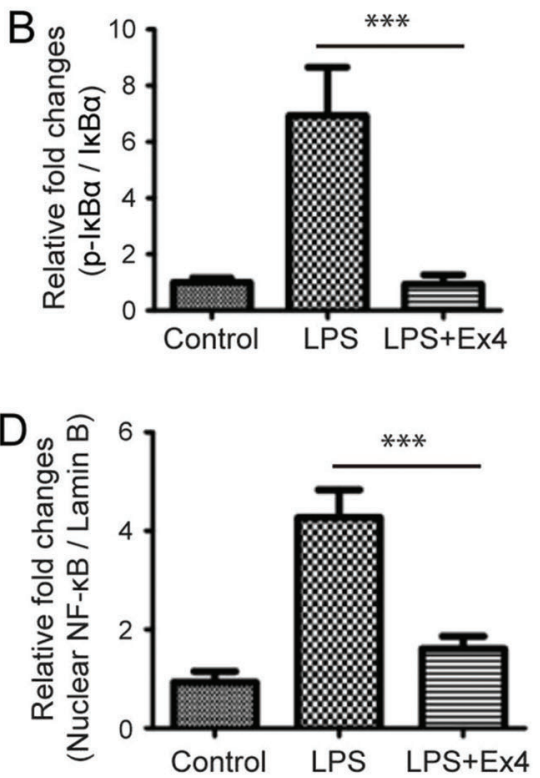

Figure 5. Effect of exendin-4 (Ex4) on lipopolysaccharide (LPS)-activated NF-кB pathway in RAW264 macrophages. RAW264 cells were treated with LPS or a combination of LPS and Ex4 for 30 min. Western blot image $(A)$ and quantification $(B)$ of basal protein and

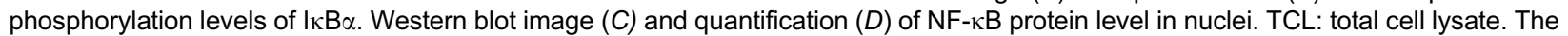
results are representative of three independent experiments. Data are reported as means $\pm S D$. ${ }^{* *} P<0.001$ (ANOVA).

\section{Discussion}

T2D represents a significant threat to global health and human life (31). Insulin resistance is an important contributor of T2D. Chronic inflammation is a major cause of insulin resistance $(27,32)$. The infiltration and accumulation of macrophages drive inflammation in the adipose tissues, and induced the secretion of inflammatory cytokines such as $T N F \alpha$, which in turn lead to adipocyte dysfunction (33). Thus, inhibition of macrophage infiltration and adipose inflammation is an important target for clinical treatment of insulin resistance and T2D.

GLP-1 is a gut hormone, which can increase pancreatic secretion of insulin and be used in the clinical therapy of T2D (16). In the current study, we found that exendin-4 showed an inhibitory effect on migration of RAW264 macrophages. This result suggests that GLP could directly target macrophages and suppress its infiltration. Consistently, previous studies have reported that GLP-1 inhibits macrophage infiltration in adipose tissue (19), liver and vessel wall (34).

Chronic inflammation plays a critical role in the development of insulin resistance. Proinflammatory cytokines are mainly secreted by inflammatory macrophages in adipose tissue (16). Our results showed that GLP-1 treatment reduced the release of the inflammatory cytokines TNF- $\alpha$, IL- 6 , and IL- $1 \beta$ in LPS-stimulated macrophages. These results are consistent with previous observations that GLP-1 inhibits the secretion of IL-1 $\beta$ and TNF- $\alpha$ in vitro (18) as well as IL-1 $\beta$ and IL-6 in vivo (35). Our observations taken together with previous studies suggest that GLP-1 has anti-inflammatory properties in macrophages. It has been indicated that proinflammatory cytokines can directly affect insulin signaling pathway and impair insulin sensitivity (36). Bouzakri and Zierath (37) reported that TNF- $\alpha$ leads to insulin resistance by directly targeting muscle insulin signaling. Accordingly, our results showed that LPS-treated macrophage CM decreased the insulin-stimulated glucose uptake in 3T3-L1 adipocytes, and this effect was reversed by $\mathrm{CM}$ from macrophages treated with exendin-4. These results suggest that GLP-1 increases insulin sensitivity by inhibiting the production of inflammatory cytokines in macrophages.

It has been well documented that activation of NF- $\mathrm{KB}$ plays a central role in inflammatory events $(12,14)$. Our results showed that GLP-1 inhibited the activation of NF- $\kappa \mathrm{B}$ pathway in macrophages, which is consistent with a previous in vivo study (19). Another study demonstrated that the main effects of GLP-1 are regulated by the activation of adenylate cyclase and the production of cAMP (38). Meanwhile, cAMP/PKA pathway regulates inflammatory response of macrophages via inhibiting the production of proinflammatory cytokines $(39,40)$. Arakawa et al. have indicated that LPS-induced macrophage activation and TNF- $\alpha$ expression was significantly reduced by GLP-1 analog exendin-4 through PKA/NF- $\mathrm{KB}$ signaling pathway (20). Moreover, It has been reported that activation of NF- $\kappa B$ can stimulate the transcription of proinflammatory genes 

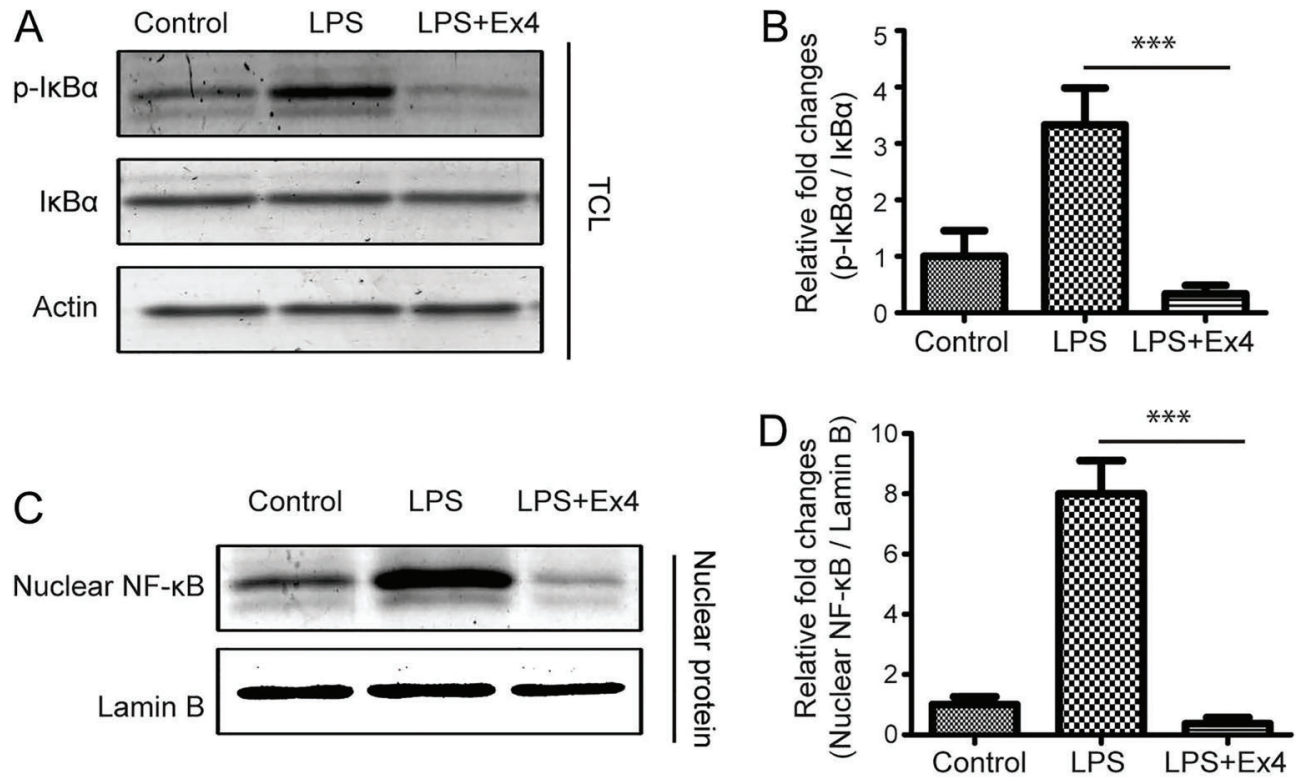

Figure 6. Effect of exendin-4 (Ex4) on lipopolysaccharide (LPS)-activated NF-kB pathway in mouse peritoneal macrophage. Mouse peritoneal macrophages were treated with LPS or a combination of LPS and Ex4 for $30 \mathrm{~min}$. Western blot image $(A)$ and quantification $(B)$ of basal protein and phosphorylation levels of $I \kappa B \alpha$. Western blot image $(C)$ and quantification $(D)$ of NF-kB protein level in nuclei. TCL: total cell lysate. The results are representative of three independent experiments. Data are reported as means $\pm S D$. ${ }^{* * *} \mathrm{P}<0.001$ (ANOVA).

including TNF- $\alpha$, IL-1 $\beta$, IL-6, and IL-8 (14). Taken together, our results and previous observations suggest that GLP-1 inhibits inflammatory response of macrophages and the production of TNF- $\alpha$, IL- 6 , and IL- $1 \beta$ by $\mathrm{CAMP} / \mathrm{PKA} / \mathrm{NF}-\mathrm{KB}$ signaling pathway.

Although the improving effect of GLP-1 on insulin resistance by targeting inflammatory macrophages was demonstrated in this study, only RAW246 macrophage cell line, mouse peritoneal macrophages and 3T3-L1 adipocytes were used in vitro. Therefore, there are some limitations in our study. In future researches, whether GLP-1 inhibits inflammatory response of macrophages and therefore

\section{References}

1. Kraakman MJ, Murphy AJ, Jandeleit-Dahm K, Kammoun $\mathrm{HL}$. Macrophage polarization in obesity and type 2 diabetes: weighing down our understanding of macrophage function? Front Immunol 2014; 5: 470, doi: 10.3389/fimmu.2014.00470.

2. Weisberg SP, McCann D, Desai M, Rosenbaum M, Leibel $\mathrm{RL}$, Ferrante AW Jr. Obesity is associated with macrophage accumulation in adipose tissue. J Clin Invest 2003; 112: 1796-1808, doi: 10.1172/JCI200319246.

3. Virtue S, Vidal-Puig A. Adipose tissue expandability, lipotoxicity and the Metabolic Syndrome - an allostatic perspective. Biochim Biophys Acta 2010; 1801: 338-349, doi: 10.1016/j.bbalip.2009.12.006.

4. Brown BN, Ratner BD, Goodman SB, Amar S, Badylak SF. Macrophage polarization: an opportunity for improved improves insulin resistance in vivo needs to be further investigated.

In conclusion, our findings suggest that GLP-1 analogue had inhibitory effects on macrophage-mediated adipose tissue inflammation and could be used for therapy in patients with T2D.

\section{Acknowledgements}

This work was supported by the National Science Foundation of China (No. 81000356) and Zhejiang Provincial Natural Science Foundation (LY16H070006). outcomes in biomaterials and regenerative medicine. Biomaterials 2012; 33: 3792-3802, doi: 10.1016/j.biomaterials. 2012.02.034.

5. Mantovani A, Sica A, Sozzani S, Allavena P, Vecchi A, Locati $M$. The chemokine system in diverse forms of macrophage activation and polarization. Trends Immunol 2004; 25: 677-686, doi: 10.1016/j.it.2004.09.015.

6. Gordon S, Taylor PR. Monocyte and macrophage heterogeneity. Nat Rev Immunol 2005; 5: 953-964, doi: 10.1038/ nri1733.

7. Fujisaka S, Usui I, Bukhari A, Ikutani M, Oya T, Kanatani Y, et al. Regulatory mechanisms for adipose tissue M1 and M2 macrophages in diet-induced obese mice. Diabetes 2009; 58: 2574-2582, doi: 10.2337/db08-1475. 
8. Fujisaka S, Usui I, Kanatani Y, Ikutani M, Takasaki I, Tsuneyama K, et al. Telmisartan improves insulin resistance and modulates adipose tissue macrophage polarization in high-fat-fed mice. Endocrinology 2011; 152: 1789-1799, doi: 10.1210/en.2010-1312.

9. Manna P, Kalita J. Beneficial role of vitamin K supplementation on insulin sensitivity, glucose metabolism, and the reduced risk of type 2 diabetes: A review. Nutrition 2016; 32 : 732-739, doi: 10.1016/j.nut.2016.01.011.

10. Lumeng CN, Bodzin JL, Saltiel AR. Obesity induces a phenotypic switch in adipose tissue macrophage polarization. J Clin Invest 2007; 117: 175-184, doi: 10.1172/JCI29881.

11. Sun K, Kusminski CM, Scherer PE. Adipose tissue remodeling and obesity. J Clin Invest 2011; 121: 2094-2101, doi: 10.1172/ JCl45887.

12. Baldwin AS Jr. The NF-kappa B and I kappa B proteins: new discoveries and insights. Annu Rev Immunol 1996; 14 : 649-683, doi: 10.1146/annurev.immunol.14.1.649.

13. Hunter JE, Butterworth JA, Zhao B, Sellier H, Campbell KJ, Thomas HD, et al. The NF-kappaB subunit c-Rel regulates Bach2 tumour suppressor expression in B-cell lymphoma. Oncogene 2016; 35: 3476-3484, doi: 10.1038/ onc.2015.399.

14. Tak PP, Firestein GS. NF-kappaB: a key role in inflammatory diseases. J Clin Invest 2001; 107: 7-11, doi: 10.1172/ $\mathrm{JCl} 11830$.

15. Aupperle KR, Bennett BL, Boyle DL, Tak PP, Manning AM, Firestein GS. NF-kappa B regulation by I kappa B kinase in primary fibroblast-like synoviocytes. J Immunol 1999; 163: 427-433.

16. Hogan AE, Gaoatswe G, Lynch L, Corrigan MA, Woods C, O'Connell J, et al. Glucagon-like peptide 1 analogue therapy directly modulates innate immune-mediated inflammation in individuals with type 2 diabetes mellitus. Diabetologia 2014; 57: 781-784, doi: 10.1007/s00125-013-3145-0.

17. Vilsboll T, Krarup T, Sonne J, Madsbad S, Volund A, Juul $A G$, et al. Incretin secretion in relation to meal size and body weight in healthy subjects and people with type 1 and type 2 diabetes mellitus. J Clin Endocrinol Metab 2003; 88: 2706-2713, doi: 10.1210/jc.2002-021873.

18. Buldak L, Machnik G, Buldak RJ, Labuzek K, Boldys A, Belowski D, et al. Exenatide (a GLP-1 agonist) expresses anti-inflammatory properties in cultured human monocytes/ macrophages in a protein kinase $A$ and B/Akt manner. Pharmacol Rep 2016; 68: 329-337, doi: 10.1016/j.pharep. 2015.10.008.

19. Lee YS, Park MS, Choung JS, Kim SS, Oh HH, Choi CS, et al. Glucagon-like peptide-1 inhibits adipose tissue macrophage infiltration and inflammation in an obese mouse model of diabetes. Diabetologia 2012; 55: 2456-2468, doi: 10.1007/s00125-012-2592-3.

20. Arakawa M, Mita T, Azuma K, Ebato C, Goto H, Nomiyama $\mathrm{T}$, et al. Inhibition of monocyte adhesion to endothelial cells and attenuation of atherosclerotic lesion by a glucagon-like peptide-1 receptor agonist, exendin-4. Diabetes 2010; 59: 1030-1037, doi: 10.2337/db09-1694[pii].

21. Yang $Y$, Tong $Y$, Gong M, Lu Y, Wang C, Zhou M, et al. Activation of PPARbeta/delta protects pancreatic beta cells from palmitate-induced apoptosis by upregulating the expression of GLP-1 receptor. Cell Signal 2014; 26: 268278, doi: 10.1016/j.cellsig.2013.11.019.
22. Gupta NA, Mells J, Dunham RM, Grakoui A, Handy J, Saxena NK, et al. Glucagon-like peptide-1 receptor is present on human hepatocytes and has a direct role in decreasing hepatic steatosis in vitro by modulating elements of the insulin signaling pathway. Hepatology 2010; 51: 1584-1592, doi: 10.1002/hep.23569.

23. Wang $H$, Wang $L$, Song $L$, Zhang $Y W, Y e J, X u R X$, et al. TNNI3K is a novel mediator of myofilament function and phosphorylates cardiac troponin I. Braz J Med Biol Res 2013; 46: 128-137, doi: 10.1590/1414-431X20122515.

24. Wang L, Wang $H$, Ye J, Xu RX, Song L, Shi N, et al. Adenovirus-mediated overexpression of cardiac troponin I-interacting kinase promotes cardiomyocyte hypertrophy. Clin Exp Pharmacol Physiol 2011; 38: 278-284, doi: 10.1111/j.1440-1681.2011.05499.x.

25. Wang $H$, Chen $Y$, Lu XA, Liu G, Fu Y, Luo Y. Endostatin prevents dietary-induced obesity by inhibiting adipogenesis and angiogenesis. Diabetes 2015; 64: 2442-2456, doi: $10.2337 / \mathrm{db} 14-0528$.

26. Kwon DY, Kim YS, Ryu SY, Choi YH, Cha MR, Yang HJ, et al. Platyconic acid, a saponin from Platycodi radix, improves glucose homeostasis by enhancing insulin sensitivity in vitro and in vivo. Eur $J$ Nutr 2012; 51: 529-540, doi: 10.1007/s00394-011-0236-x.

27. Wang P, Mariman E, Renes J, Keijer J. The secretory function of adipocytes in the physiology of white adipose tissue. $J$ Cell Physiol 2008; 216: 3-13, doi: 10.1002/jcp.21386.

28. Edwards CM, Stanley SA, Davis R, Brynes AE, Frost GS, Seal LJ, et al. Exendin-4 reduces fasting and postprandial glucose and decreases energy intake in healthy volunteers. Am J Physiol Endocrinol Metab 2001; 281: E155-E161, doi: 0193-1849/01.

29. Temelkova-Kurktschiev T, Siegert G, Bergmann S, Henkel E, Koehler C, Jaross W, et al. Subclinical inflammation is strongly related to insulin resistance but not to impaired insulin secretion in a high risk population for diabetes. Metabolism 2002; 51: 743-749, doi: S002604950242029X.

30. Festa A, Hanley AJ, Tracy RP, D’Agostino R Jr, Haffner SM. Inflammation in the prediabetic state is related to increased insulin resistance rather than decreased insulin secretion. Circulation 2003; 108: 1822-1830, doi: 10.1161/01.CIR.000 0091339.70120.53.

31. Tuomilehto J, Lindstrom J, Eriksson JG, Valle TT, Hamalainen $\mathrm{H}$, llanne-Parikka $\mathrm{P}$, et al. Prevention of type 2 diabetes mellitus by changes in lifestyle among subjects with impaired glucose tolerance. N Engl J Med 2001; 344: 1343-1350, doi: 10.1056/ NEJM200105033441801.

32. Scherer PE. Adipose tissue: from lipid storage compartment to endocrine organ. Diabetes 2006; 55: 1537-1545, doi: $10.2337 / \mathrm{db06}-0263$.

33. Chen XH, Zhao YP, Xue M, Ji CB, Gao CL, Zhu JG, et al. TNF-alpha induces mitochondrial dysfunction in 3T3-L1 adipocytes. Mol Cell Endocrinol 2010; 328: 63-69, doi: 10.1016/ j.mce.2010.07.005.

34. Wang Y, Parlevliet ET, Geerling JJ, van der Tuin SJ, Zhang $\mathrm{H}$, Bieghs $\mathrm{V}$, et al. Exendin-4 decreases liver inflammation and atherosclerosis development simultaneously by reducing macrophage infiltration. $\mathrm{Br} J$ Pharmacol 2014; 171: 723-734, doi: 10.1111/bph.12490.

35. Robinson E, Cassidy RS, Tate M, Zhao Y, Lockhart $S$, Calderwood D, et al. Exendin-4 protects against 
post-myocardial infarction remodelling via specific actions on inflammation and the extracellular matrix. Basic Res Cardiol 2015; 110: 20, doi: 10.1007/s00395-015-0476-7.

36. Lang J. Molecular mechanisms and regulation of insulin exocytosis as a paradigm of endocrine secretion. Eur $J$ Biochem 1999; 259: 3-17, doi: 10.1046/j.1432-1327. 1999.00043.

37. Bouzakri K, Zierath JR. MAP4K4 gene silencing in human skeletal muscle prevents tumor necrosis factor-alpha-induced insulin resistance. J Biol Chem 2007; 282: 7783-7789, doi: 10.1074/jbc.M608602200.

38. Brubaker PL, Drucker DJ. Minireview: Glucagon-like peptides regulate cell proliferation and apoptosis in the pancreas, gut, and central nervous system. Endocrinology 2004; 145: 2653-2659, doi: 10.1210/en.2004-0015[pii].

39. Aronoff DM, Canetti C, Serezani CH, Luo M, Peters-Golden $M$. Cutting edge: macrophage inhibition by cyclic AMP (cAMP): differential roles of protein kinase $A$ and exchange protein directly activated by cAMP-1. J Immunol 2005; 174 : 595-599, doi: 174-2/595 [pii].

40. Mogi C, Tobo M, Tomura H, Murata N, He XD, Sato K, et al. Involvement of proton-sensing TDAG8 in extracellular acidification-induced inhibition of proinflammatory cytokine production in peritoneal macrophages. $J$ Immunol 2009; 182: $3243-3251$, doi: $10.4049 /$ jimmunol.0803466182/5/ 3243 [pii]. 